\title{
Personalization Dimensions for MHealth to Improve Behavior Change: A Scoping Review
}

\author{
Laëtitia GOSETTO $^{\mathrm{a}, 1}$, Frédéric EHRLER ${ }^{\mathrm{b}}$ and Gilles FALQUET ${ }^{\mathrm{a}}$ \\ ${ }^{a}$ University of Geneva, Switzerland \\ ${ }^{\mathrm{b}}$ University Hospitals of Geneva, Switzerland
}

\begin{abstract}
Due to the large number of smartphone users, mHealth has become a popular support to foster users' health behavior change Personalization is an important factor to increase the effectiveness of mHealth interventions. Based on a literature review, we have listed and categorized personalization concepts associated with behavior change in mHealth into 4 dimensions, users, system functionalities, information, and app properties. The users dimension refers to user-related characteristics such as personality, player profile, need for cognition and perception of social norms. The system functionalities contain the functionalities that can be found in applications such as reminders as well as gamification functionalities such as collectibles. The information dimension concerns the way information is transmitted, such as the source of the message must be expert or the type of feedback to be provided. Finally, there are app properties such as the aesthetics of the application. For the next part, it would be interesting to discover the links we can make between the dimensions.
\end{abstract}

Keywords. MHealth, Mobile, Application, Health, Behavior Change Theory, Personalization, Gamification

\section{Introduction}

MHealth can be defined as the use of mobile computing and communication technologies in health care and public health [1]. About $79 \%$ of the European population used their smartphone in 2016 to go online [2]. Smartphones possess some features, including apps text messaging, Bluetooth, and others, that can be useful to change user behavior towards healthier ones [3]. Integrating behavior change theories (BCT) is one of the popular techniques employed in mHealth. BCT, is defined by Michie et al. as "like an observable, replicable, and irreducible component of an intervention designed to alter or redirect causal processes that regulate behavior" [4]. Researchers are therefore integrating interventions such as goal setting or self-monitoring of behavior [5].

Personalization is another mechanism that can be incorporated into mHealth interventions to promote behavioral change [6]. Personalization can be defined as the incorporation of recognizable aspects of a person into tailored content, such as a person's name [7]. The importance of personalization is already widely recognized since it is

\footnotetext{
${ }^{1}$ Corresponding author, Laëtitia Gosetto, University of Geneva, Route de Drize 7, 1227 Carouge; Email: laetitia.gosetto@unige.ch
} 
found as criteria in many rating scales for mobile health applications such as the Mobile Application Rating Scale (MARS) [8], or the App Behavior Change Scale (ABACUS) [9].

Since personalization in mHealth can be applied in many ways, from simply adding the user's name to adapting the content to the user's personality, one may ask on which dimensions can we customize?

The purpose of this article is to present the different dimensions of personalization of mHealth intervention to promote behavioral change based on a review of the literature.

\section{Method}

Searches were conducted on the ScienceDirect, ResearchGate online databases for articles from 2008 to 2020.2008 being the release of the first smartphone and thus the current mHealth. The selected articles had to treat personalization and applications for behavioral change as well as the evaluation of applications for behavior change. They also had to be written in English.

The terms used for the search were: personalization, mHealth, gamification, personality, tailoring, app features, app functionality, scale app mHealth, guideline app. A first selection was made base on the reading of the titles and abstracts. Then, a second reading of the full article allowed to determine if the article met the eligibility criteria. We have then listed the personalization techniques found in these articles.

We have also listed the features that appear in different scales used to assess application for behavior change. We have selected four scales, the MARS [8], the ABACUS [9], the persuasive system design [10] and the ergonomic criteria grid for the assessment of ergonomic persuasion [11].

All concepts were organized into a conceptual map helping us to regroup them into several dimensions.

\section{Results}

1825 articles were extracted, and 27 articles met the eligibility criteria. From the 27 articles, we were able to extract a list of concepts that are used to personalize intervention. From this list, we organized them into a conceptual map and group them into four dimensions.

\subsection{Definition of Dimensions}

The literature presents 39 personalization concepts, ranging from the personality of the user to the characteristics of the messages. We have organized these personalization concepts into a concept map summarized in the form of a table (see Table 1). This helped us to identify several dimensions to characterize the personalization concepts. We defined 4 dimensions: user, system functionalities, information, and app properties. These dimensions are detailed below. We identified 39 concepts, 5 for the dimension user, 17 for the dimension system functionalities, 13 for the dimension information and 4 for the dimension app properties. We also indicated which concepts were present in the four scales used to assess application for behavior change. 


\subsubsection{User}

This dimension contains all user-specific characteristics that can be used for personalization. The literature shows links between user personality and gaming characteristics [12]. Personality is measured using the Big Five [13], a model with five factors neuroticism, openness, conscientiousness, altruism, and extroversion, defining personality.

The profile of the players is another user characteristic for personalization of mHealth intervention derived from gamification theory. Several scales exist to define the user's type of player, as well as his preferences for the games. We have chosen two scales for this representation, Tondello's Hexad Scale [14] and the taxonomy of player motivation by Yee [15]. Each one defines a type of player and the type of games or interaction he prefers. We chose these scales because according to the literature, there would be a link between the type of player and the gamification features [14][15]. For example, according to the Hexad Scale, philanthropists are motivated by a goal, are altruistic and willing to give without expecting a reward. It is therefore necessary to incorporate elements of collection and exchange into the game to appeal to this type of user. [14].

Another interesting feature is the need for cognition [16]. This characteristic defines people according to their individual differences in intrinsic motivation to engage in effortful cognitive endeavors [16],[17]. It may be interesting to consider this characteristic, as for example, individuals with high need-for-cognition are more influenced by quality messages while low need-for-cognition are more influenced by peripheral cues [18].

Finally, the last characteristic we have integrated is the perception of the subjective norm. This characteristic is common to many theories of behavior change, such as the Theory of planned Behavior [19] or the Integrated Behavior Mode [20]. This characteristic refers to the perceived social pressure to perform or not to perform the behavior. As a general rule, the more the subjective norm is in agreement with the behavior, the more the individual will intend to change behavior in accordance with this subjective norm [19].

\subsubsection{System Functionalities}

In this dimension we included the functionalities of applications that can be personalized according to the literature. Functionalities refer to the services the application provides to the user, such as reminders or self-monitoring. Self-monitoring as "occurring when an individual first self-assesses whether or not a target behavior has occurred, and then self-records the occurrence, frequency, duration, or so on of the target behavior"'[21].

We have also included gaming features that can be personalized. Such as goal setting, rewards or levels and progression.

\subsubsection{Information}

This dimension groups together characteristics that are related to the transmission of information in an application. One part concerns the knowledge and information to be transmitted, such as the importance of relying on an expert source to provide the content, 
or to provide basic information about the desired behavior. These characteristics are extracted from different scales such as MARS [8] or ABACUS [9].

Another part concerns feedback. Feedback consists in presenting individuals with information about themselves, obtained through the application. There are 3 types of feedback, descriptive (provides only a description of the user's behavior in relation to his data), evaluative (provides an interpretation based on the user's behavior) and comparative (provides feedback comparing the user with other people). Each may be more or less effective depending on the user. For example, comparative feedback will work best for a person who needs to have a high level of social norms [22].

\subsubsection{App Properties}

The App properties dimension regroups features that are specific to mobile applications. In particular, it includes the aesthetic features, extracted from the MARS scale [8]. As well as one feature, customization. Customization means that "the user explicitly states interests and preferences through direct configuration of human-computer interfaces (HCI), system's options or screens" [23].

Table 1. Representation of the dimension to personalize mHealth

\begin{tabular}{|c|c|c|c|}
\hline Users & $\begin{array}{l}\text { System } \\
\text { Functionalities }\end{array}$ & Information & App Properties \\
\hline $\begin{array}{l}\text { Personality (e.g Big- } \\
\text { Five) }[12][13]\end{array}$ & $\begin{array}{l}\text { App Functionalities } \\
\text { (e.g reminder, self- } \\
\text { monitoring) })^{* *}[21]\end{array}$ & $\begin{array}{l}\text { Knowledge and } \\
\text { information (e.g. expert } \\
\text { source, quantity of } \\
\text { information) } * * * *[8][9]\end{array}$ & $\begin{array}{l}\text { Aesthectics (Layout, } \\
\text { visual appeal, } \\
\text { graphics)* [8] }\end{array}$ \\
\hline $\begin{array}{l}\text { Gamer Profil (e.g } \\
\text { Hexad scale) }[14][15]\end{array}$ & $\begin{array}{l}\text { Gamification Features } \\
\text { (e.g Rewards, } \\
\text { cooperation)**** } \\
{[12][14][15]}\end{array}$ & $\begin{array}{l}\text { Feedbacks (evaluative, } \\
\text { descriptive, } \\
\text { comparative)* }{ }^{* 22]}\end{array}$ & $\begin{array}{l}\text { App Features } \\
\text { (customizable } * * * * \text { ) } \\
{[23]}\end{array}$ \\
\hline \multicolumn{4}{|l|}{$\begin{array}{l}\text { Need-for-cognition } \\
{[16][17][18]}\end{array}$} \\
\hline $\begin{array}{l}\text { Perception of the social } \\
\text { norm }[19][20]\end{array}$ & & & \\
\hline
\end{tabular}

\section{Discussion}

From the literature, we have identified and classified personalization concepts into 4 dimensions, users, system functionalities, information, and app properties. From this classification, we can identify on which characteristics it is possible to personalize. For example, what kind of feedback to provide for each user etc...

It would be interesting as a future research to study the notion of design, such as design with empathy or emotional design. In particular, emotional design has the potential to bring personality to the application in order to make it more attractive to the user. It would be also interesting to explore the relationship between the characteristics belonging to different dimensions. For example, what kind of gamification features are favored by people who fit the big-five's extroversion profile. In this way, one could personalize features of the application according to the user's personality. It would also 
be interesting to define how to obtain information about the user in order to personalize according to the other dimensions.

\section{References}

[1] Free C, Phillips G, Felix L, and others. The effectiveness of M-health technologies for improving health and health services: a systematic review protocol. BMC Res Notes. 2010;3(250):1-7.

[2] Eurostat. 2016 [cited 2020 Sept Available 17]. from: https://ec.europa.eu/eurostat/documents/2995521/7771149/9-20122016-BP-FR.pdf/7e0f0705-cabd4a1c-b57d-f893c5093947

[3] WHO. World Health Organization - mHealth New horizons for health through mobile technologies. 2011. [cited 2020 Sept 17]. Available from: https://www.who.int/goe/publications/goe_mhealth_web.pdf

[4] Michie S, Richardson M, Johnston M, and others. The Behavior Change Technique Taxonomy (v1) of 93 Hierarchically Clustered Techniques: Building an International Consensus for the Reporting of Behavior Change Interventions. Ann Behav Med. 2013;46:81-95.

[5] Direito A, Pfaeffli Dale L, Shields E, and others. Do physical activity and dietary smartphone applications incorporate evidence-based behaviour change techniques? BMC Public Health. 2014 Jun;14(1):1-7.

[6] Wanyonyi KL, Themessl-Huber M, Humphris G, Freeman R. A systematic review and meta-analysis of face-to-face communication of tailored health messages: Implications for practice. Patient Educ Couns. 2011;85(3):348-55.

[7] Dijkstra A. The Psychology of Tailoring-Ingredients in Computer-Tailored Persuasion. Soc Personal Psychol Compass. 2008;2(2):765-84.

[8] Stoyanov SR, Hides L, Kavanagh DJ, and others. Mobile App Rating Scale: A New Tool for Assessing the Quality of Health Mobile Apps. JMIR mHealth uHealth. 2015 Mar;3(1):1-10.

[9] McKay FH, Slykerman S, Dunn M. The App Behavior Change Scale: Creation of a Scale to Assess the Potential of Apps to Promote Behavior Change. JMIR mHealth uHealth. 2019;7(1):1-18.

[10] Wang Y, Eng M, Fadhil A, Lange J-P, Reiterer H. Integrating Taxonomies Into Theory-Based Digital Health Interventions for Behavior Change: A Holistic Framework. JMIR Res Protoc. 2019;8(1):1-11.

[11] Nemery A, Brangier E, Kopp S. Proposition d'une grille de critères d'analyse ergono-miques des formes de persuasion interactive. Proceedings of the IHM'2010 ; 2010 ; Luxembourg. 153-6 p.

[12] Orji R, Nacke LE, Marco C Di. Towards Personality-driven Persuasive Health Games and Gamified Systems. Proceedings of the CHI. Denver; 2017. 1015-27.

[13] Mccrae RR, Costa PT. Validation of the Five-Factor Model of Personality Across Instruments and Observers. Journal of Personality and Social Psychology. 1987;52(1):81-90.

[14] Tondello GF, Wehbe RR, Diamond L, and others. The Gamification User Types Hexad Scale. Proceeding in CHI Play'16; Austin; 2016. 229-243p.

[15] Yee N. Motivations for Play in Online Games. Cyberpsychology \& Behavior. 2006;9:772-775.

[16] Cacioppo JT, Petty RE. The Need for Cognition. J Pers Soc Psychol. 1982;42(1):116-31.

[17] Cacioppo JT, Petty RE, Feng Kao C. The Efficient Assessment of Need for Cognition. J Pers Assess. 1984; 48(3):306-7.

[18] Axsom D, Yates S, Chaiken S. Audience response as a heuristic cue in persuasion. Journal of Personality and Social Psychology. US: American Psychological Association. 1987; 53:30-40.

[19] Ajzen I. The Theory of Planned Behavior. Organizational Behavior And Human Decision Processes. 1991;50:179-211.

[20] Fishbein M, Yzer MC. Using Theory to Design Effective Health Behavior Interventions. 2003;164-83.

[21] Nelson RO, Hayes SC. Theoretical Explanations for Reactivity in Self-Monitoring. Behav Modif. 1981 Jan;5(1):3-14.

[22] Hawkins RP, Kreuter M, Resnicow K, Fishbein M, Dijkstra A. Understanding tailoring in communicating about health. Health Educ Res. 2008;23(3):454-66.

[23] Madeira RN, Germano H, Macedo P, Correia N. Personalising the user experience of a mobile health application towards patient engagement. Procedia Computer Science. 2018;141: 428-33. 